\title{
THE SALICYLATES
}

\author{
VIII. SALICYI EDEMA* \\ P. J. HANZLIK, M.D., R. W. SCOTT, M.D. \\ AND \\ J. L. REYCRAFT, M.D. \\ CLEVELAND
}

Following the administration of full therapeutic doses of salicylate there is a rather marked diminution in urine output, reaching its greatest depression about ten to twenty hours after the symptoms of toxicity appear and persisting for about forty to seventy hours after the administration of the drug. The output of urine reaches its previous level roughly about the time excretion of salicyl is completed.

Two possible explanations are suggested for this: (1) sweating; (2) retention of water, that is, edema; the important factors to be considered in retention are $(a)$ tissues, and $(b)$ kidney.

It is the object of this communication to report certain facts bearing on these causes. The work was conducted in a quantitative way and on persons some of whom were practically normal, others convalescent from various disorders. The following procedures were carried out before and after the administration of the salicylate, and throughout the experiment, which usually lasted about a week. The persons were weighed regularly, about three times in ten hours, and the drug was not administered until the weight curve became practically constant. This was reached usually within two to three days after the person was put to bed and placed on the regular routine. The water and dietary intakes were maintained as constant as possible throughout the procedure. Two hundred c.c. of water were administered every two hours. Coffee or milk was substituted for this at meal times. The meals were taken at the same hours, and the quantity was constant throughout. The administration of salicyl was so timed that the symptoms of toxicity would come when the stomach would be empty so as to avoid loss of contents in case emesis should occur. No attention was paid to the qualitative nature of the dietary, but even in this there were no radical changes, since the food in this hospital is remarkably constant in quality at all times.

* Submitted for publication April 14, 1917.

* From the Pharmacological Laboratory, Western Reserve University and the Medical Clinic, City Hospital, Cleveland.

* This investigation was supported, in part, by a grant from the Therapeutic Research Committee of the Council on Pharmacy and Chemistry of the American Medical Association. 
Renal function was studied by the output of urine, which was collected in ten-hour periods; the quantitative excretion of albumin (method of Folin and Denis ${ }^{1}$ ); and daily observations on the phenolsulphonephthalein excretion (two-hour, and carried out in the usual way) and urea nitrogen of the blood (by the urease, aeration and colorimetric procedure). Hemoglobin estimations were made with the idea of ascertaining the chief depot of the water retention, that is, whether the blood or tissues. For this, the carbon monoxid colorimetric method of Haldane ${ }^{2}$ was chosen, using the Duboscq colorimeter for making the readings instead of test tubes. The results are expressed as relative percentages of hemoglobin. The effect on and the rôle of the tissues were also judged, in part by the course of salicyl excretion in the urine. The salicyl was estimated according to a method previously described. ${ }^{3}$ Sweating was judged, in part, from the weight curve and, in part, by direct inspection and the subjective symptoms reported by the patient himself.

When the weight and urine curves became practically constant, the salicylate was administered. A definite quantity (20 c.c.) of sodium salicylate (usually about 10 per cent.) was administered together with 80 c.c. of water every hour until toxicity, when the administration was stopped. The water intake was then maintained at the rate of 200 c.c. every two hours, and the various observations according to the procedures detailed above were carried out until the end of the experiment. In all, nine persons have been studied. Four of these (Patients $27,28,29$ and 30 ) received sodium bicarbonate together with the salicylate. The detailed protocols for each individual are so long that they have been omitted entirely, and instead the clata are presented in the form of curves for each one. Various clinical data pertaining to all the individuals are presented in the accompanying table.

1. Salicylate Causes Edema. - This is indicated by the fact that there is an increase in body weight demonstrable after the administration of the drug, unless this is prevented by sweating. Charts $1,2,3$, 4, 6, 7 and 9 indicate definite increases in body weight and above the level before the drug was administered. This increase ranges from moderate to considerable. The diaphoresis ranged from imperceptible to just perceptible, and roughly, inversely proportional to changes in the body weight. That is, a relatively small diaphoresis is accompanied by a more marked increase in body weight.

Charts 5 and 8 show practically no changes in body weight. These persons gave evidences of marked diaphoresis. So far as other phe-

1. Folin and Denis: Jour. Biol. Chem., 1914, 18, 273.

2. Haldane: Jour. Physiol., 1901, 26, 497.

3. Thoburn and Hanzlik: Jour. Biol. Chem., 1915, 23. 163. 


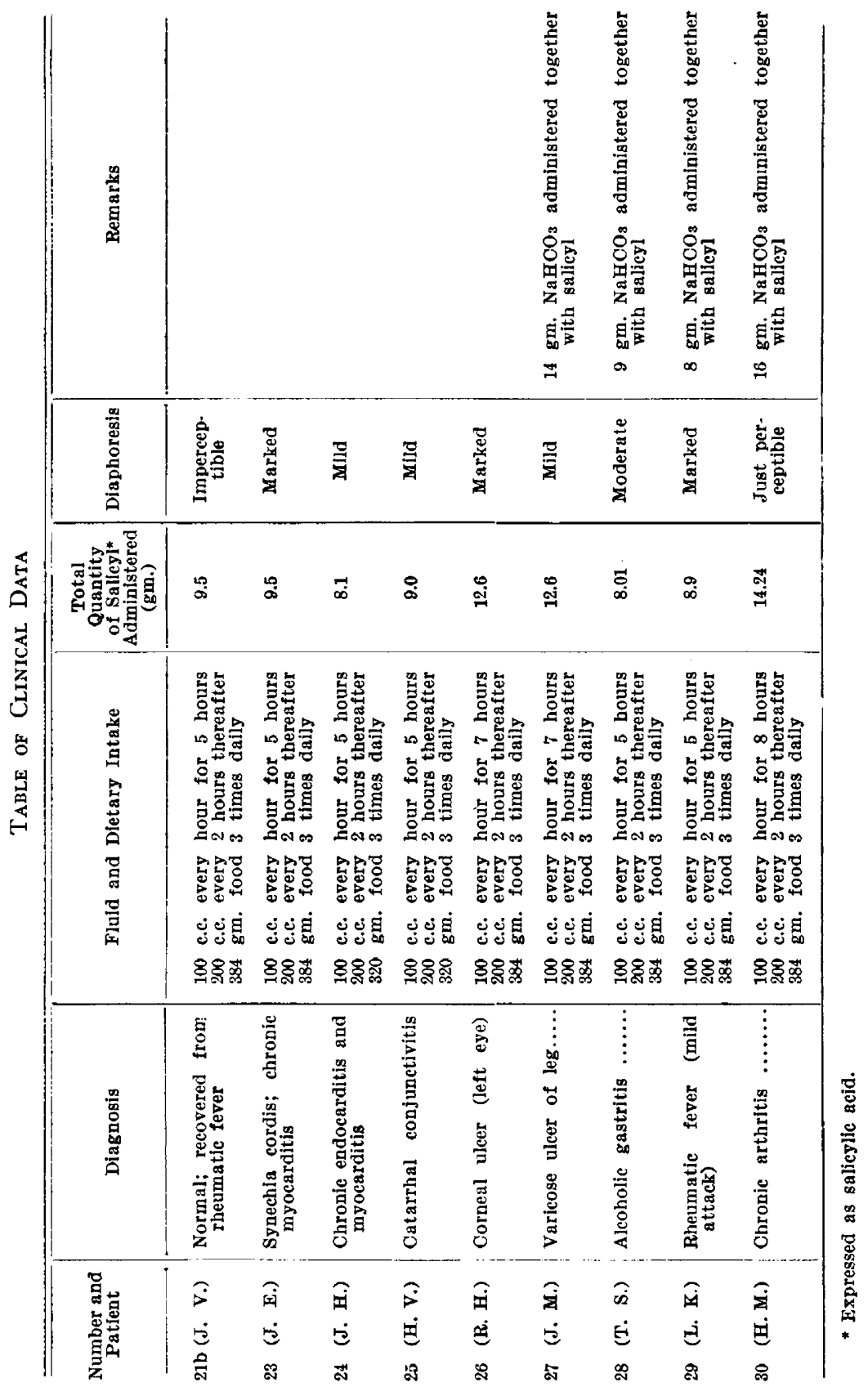




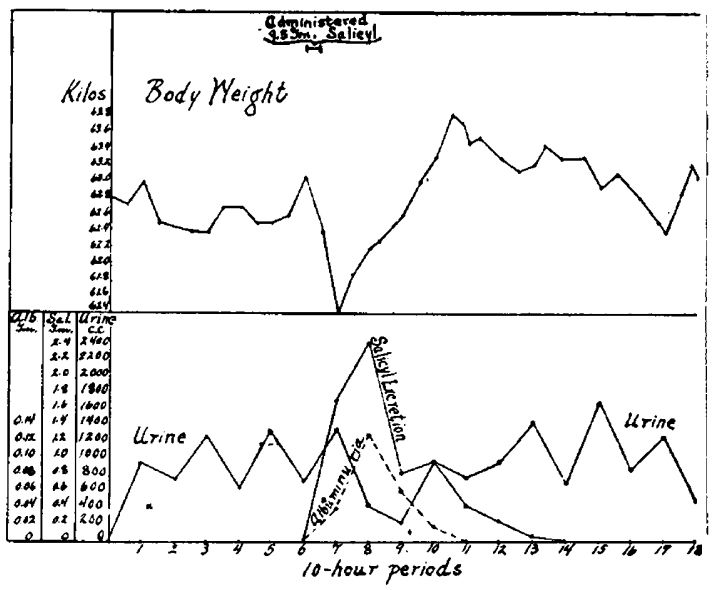

Chart 1.-Patient 21b (J. V.) In this and all the other charts the brace at the top of the chart indicates the period during which salicyl was administered; salicyl refers to salicylic acid; Alb, to albumin; sal, to salicyl; the cross $\times$, to the quantity (grams) of feces voided; 'phthalein refers to phenolsulphonephthalein.

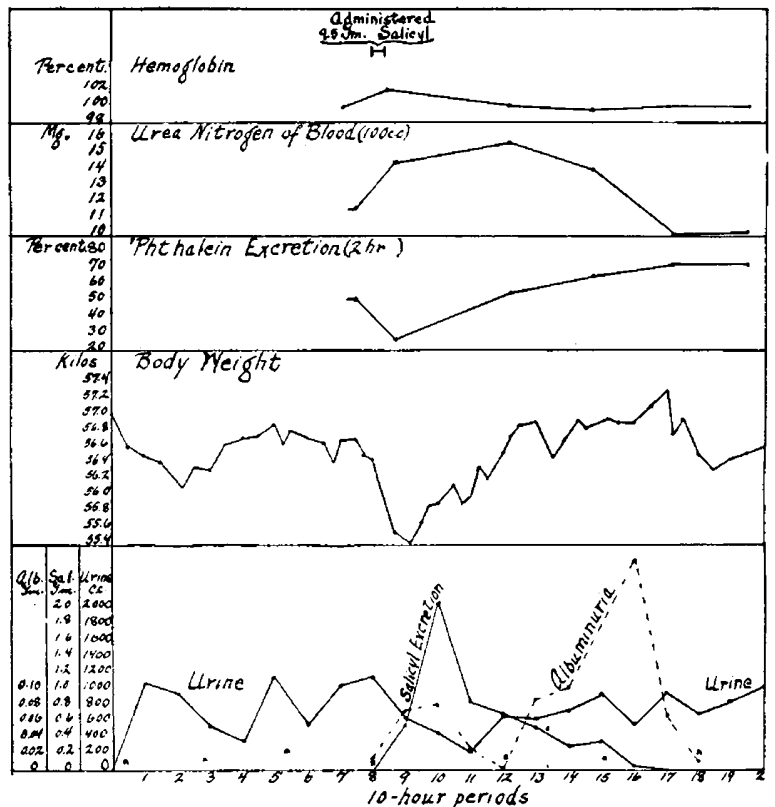

Chart 2.-Patient 23 (J. E.). Marked diaphoresis occurred from end of ninth to twelfth periods inclusive. 


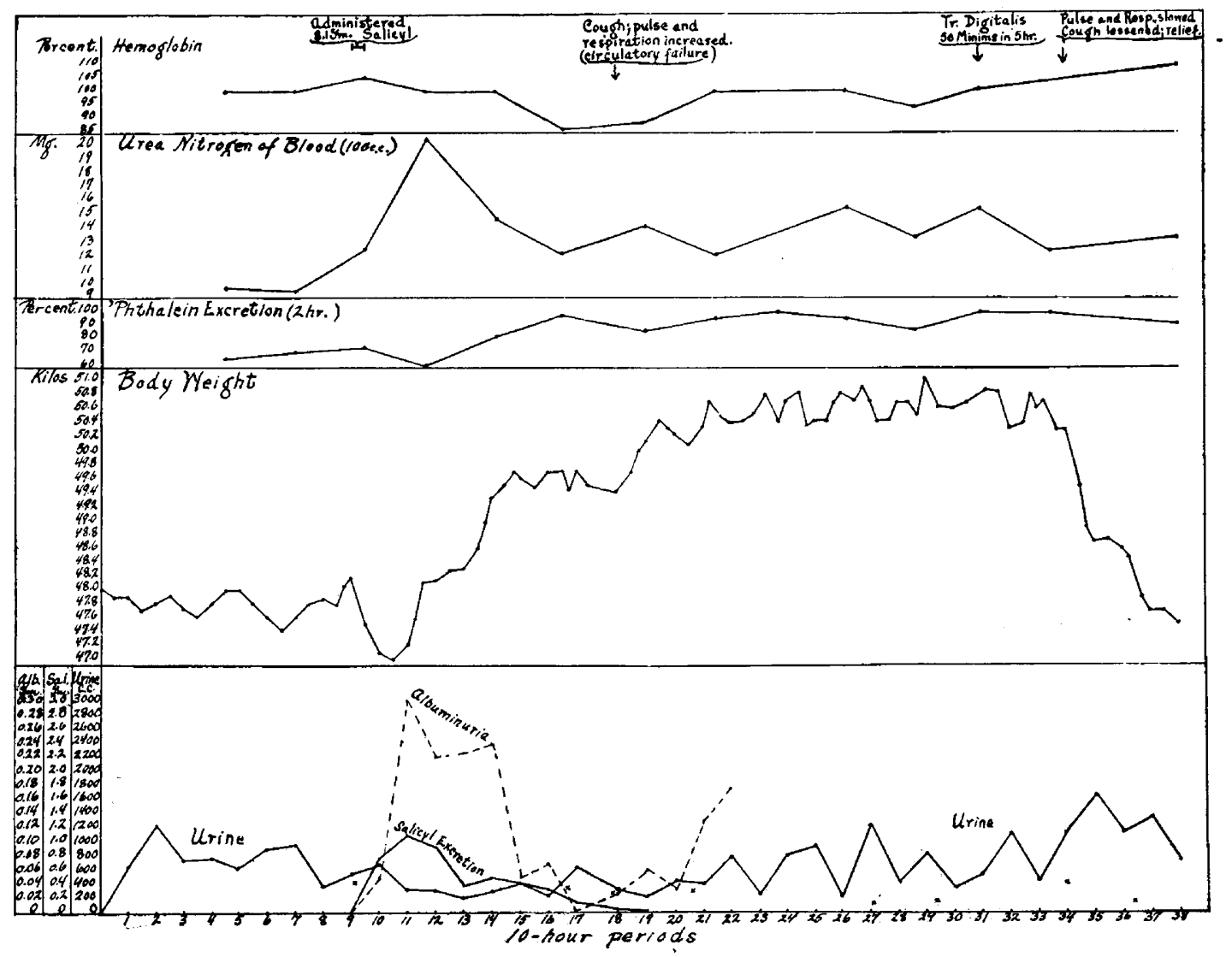

Chart 3.-Patient 24 (J. H.). Aside from the effects produced by the salicylate, this particular case presents an interesting study of digitalis action in circulatory embarrassment. About the time of appearance of certain objective symptoms, namely, increase in pulse rate, respiration and coughing, etc., there was demonstrable a dilution of the blood as indicated by a fall in the percentage of hemoglobin, a diminution in the urea content of the blood, an increase in body weight and a diminution in water output. Gradually the blood became more concentrated because the tissues now became the chief reservoir for the surplus fluid. This is indicated also by the persistence of the edema. Renal function became variable as indicated by the variable excretion of phenolsulphonephthalein and urea content of the blood, the albuminuria at the same time being markedly increased. In the meantime the only subjective symptom was a slight sensation of oppression in the chest. On inspection there was some slight fulness of the face, but otherwise edema was not demonstrable. These effects had now lasted for about 160 hours. The question arose as to whether the marked increase in body weight was due to improved nutrition, in part or whole, or anasarca resulting from circulatory embarrassment. This was tested out by the administration of tincture of digitalis, the result being a prompt and marked fall in body weight to the original level with a marked increase in urine output, improvement of renal function and some concentration of the blood. The various effects are strikingly shown in the curves of the chart. It was concluded, therefore, that the increase in body weight was due to anasarca as the result of circulatory embarrassment. 


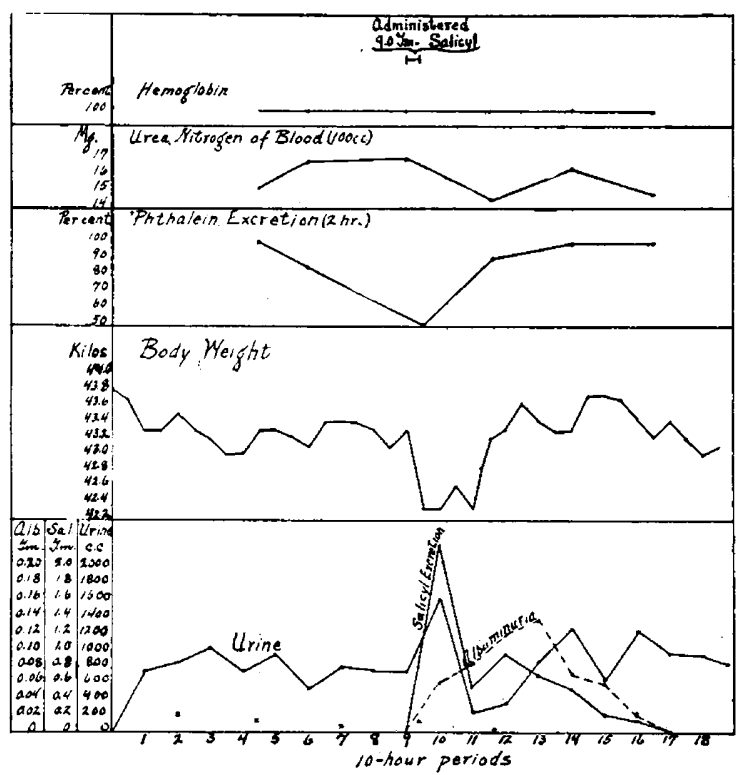

Chart 4.-Patient 25 ( $\mathrm{H}$. V.). Mild diaphoresis occurred from end of eleventh to thirteenth periods inclusive.

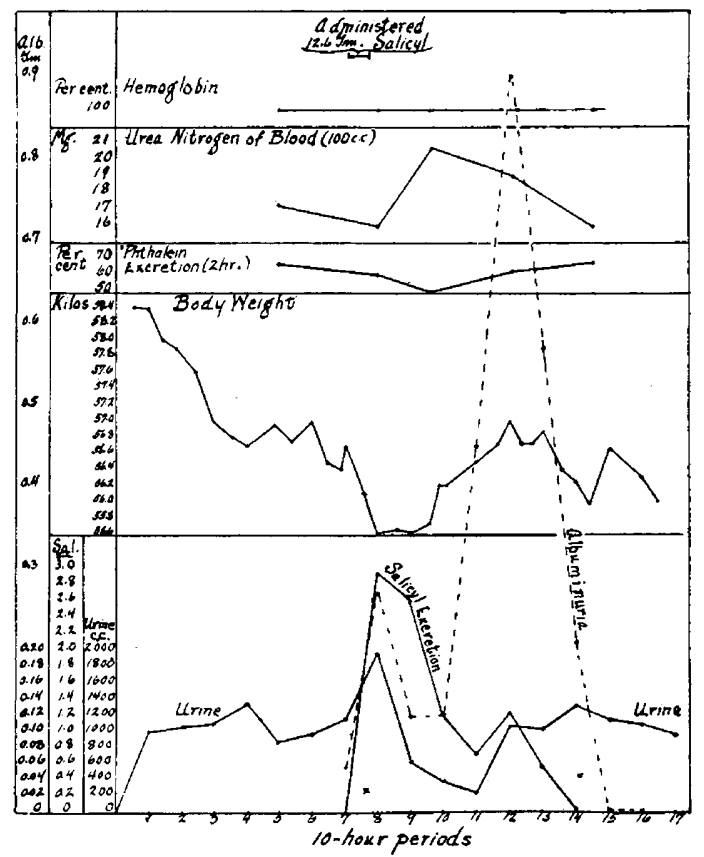

Chart 5.-Patient 26 (R. H.). Marked diaphoresis occurred from the ninth to end of eleventh periods inclusive. 
nomena are concerned, these remained unchanged. In nearly every case there is evidence of a sharp decline in body weight about the time of toxicity. This is due, no doubt, to diaphoresis which occurs at this time and when the concentration of the drug in the tissues is at its maximum. This, of course, is a well known action of salicylate. Persistent sweating in those persons who habitually sweat considerably resulted in no modification of the curves of body weight. The loss of body weight cannot be attributed to increased urine or fecal output,

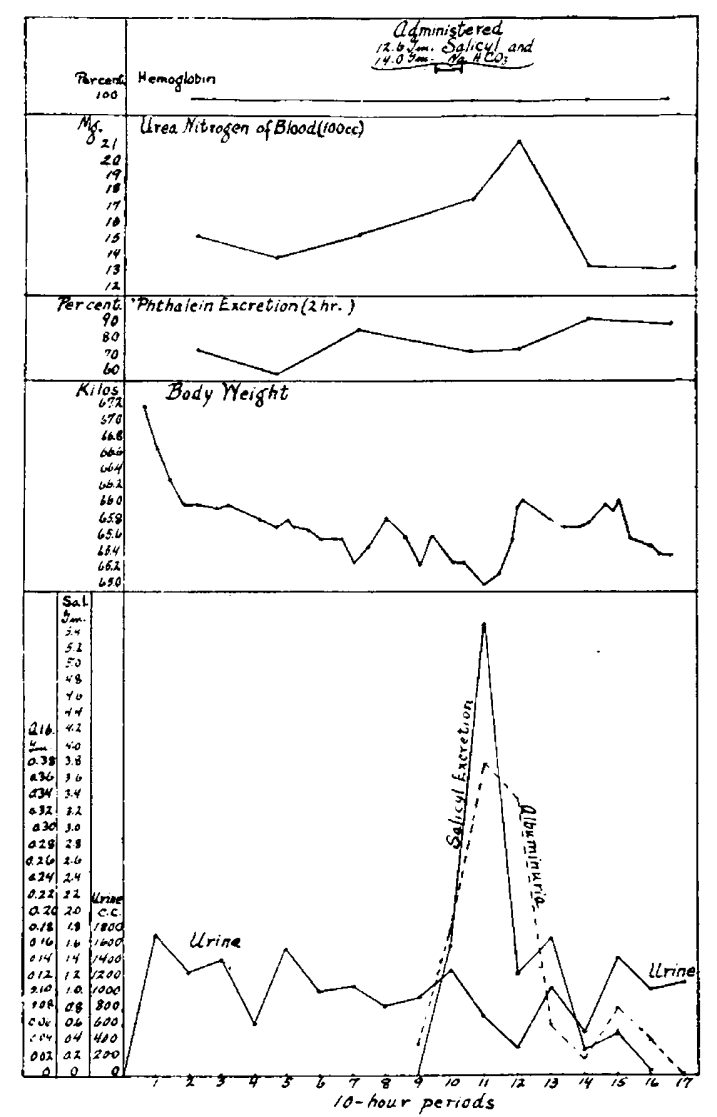

Chart 6.-Patient 27 (J. M.)

for these do not adequately compensate for the losses. The respiratory rate remained unchanged. The loss of water, therefore, could only have taken place by evaporation, that is, by diaphoresis.

It may be concluded that the administration of salicylate in fult therapeutic doses causes a relative anuria, and this is due to retention of the water, that is, edema, as indicated by an increase in body weight unless modified by sweating. 
2. The Water Retention is not Due to Retention of Salicyl in the Tissues.-This is indicated by the curves of salicyl excretion ${ }^{4}$ in all the figures, which represent the data obtained for different individuals. It is seen that the maximum excretion of salicyl occurs at a time when the body weight begins to increase and when the water excretion is lowest. The excretion is at its minimum when the body weight is at its maximum; that is, when the water retention or edema is most marked, and in most individuals this was completed before the body weight reached its previous level. The excretion of salicyl does not

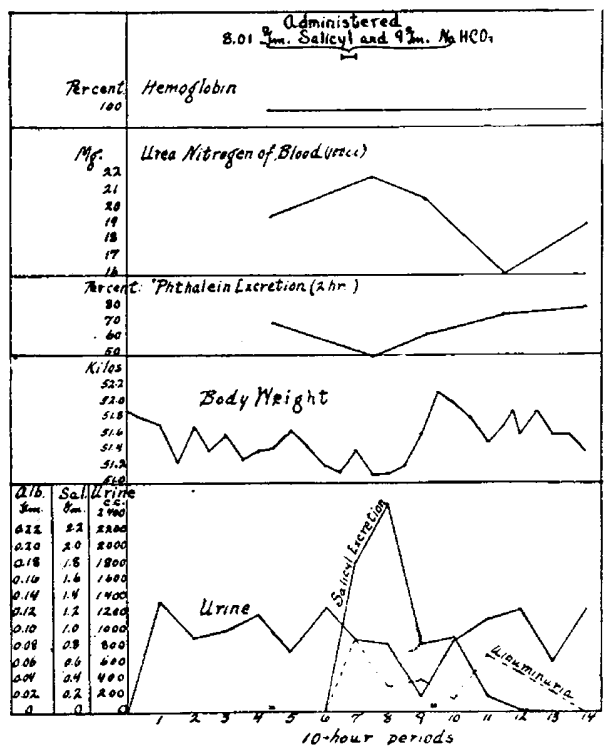

Chart 7.-Patient 28 (T. S.)

go parallel with the curve of body weight. There is no retention of salicyl and the retention of water, therefore, is not associated with or due to retention of salicyl in the tissues.

3. The Water is not Retained by the Blood.-This is indicated by the curves of hemoglobin percentage of the blood. Dilution of the blood was demonstrable in only one patient (24, Chart 3$)$, in whom for a time there was some cardiac decompensation. On the other hand, an increase in the percentage of hemoglobin at toxicity was demonstrable in two patients. These persons sweated considerably from which it is inferred that there was some concentration of the blood at this time.

4. For other studies on the quantitative excretion of salicyl consult an earlier publication by Hanzlik, Scott and Thoburn: Jour. Pharmacol. and Exper. Therap., 1917, 9, 247, and in connection with retention, Jour. Pharmacol. and Exper. Therap., 1917, 9, 217. 
In all other patients, whose blood was studied, no changes in blood volume were demonstrable.

From these evidences it appears logical to conclude that the water is retained in the tissues.

4. The Retention is Chiefly of Renal Origin. - Previous studies ${ }^{3}$ on renal function showed that the administration of salicylate impairs the functional efficiency of the kidney. This also is the case in the

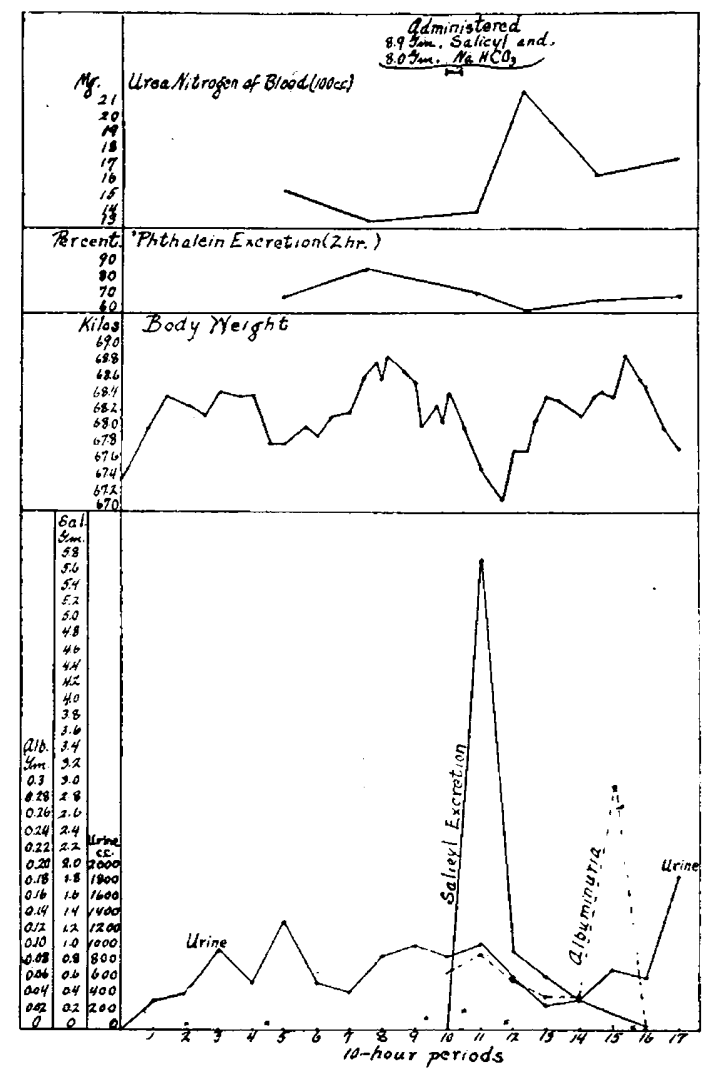

Chart 8.-Patient 29 (L. K.)

persons studied in connection with edema. Indeed it is one of the most constant features of the experiments, and is indicated in the various curves by a diminution in phenolsulphonephthalein excretion and at the same time an accumulation of urea nitrogen of the blood. Albumin, leukocytes and casts also appeared in the urines. This diminution in renal functional efficiency is demonstrable about the time of toxicity,

5. Hanzlik and Karsner: The Archives Int. Med., 1917, 19, 1016. Hanzlik, Scott and Thoburn: Ibid., 1030. 
gradually becomes more severe, and finally (end of eighty to ninety hours) returns to its previous state. In almost every instance the impairment in renal function appeared before an increase in body weight was demonstrable. From this it seems that the renal factor plays an important rôle in the production of edema. It must be admitted that the part which tissues in general might play have not been absolutely excluded, for it is conceivable that in the beginning a marked diaphoresis could compensate for any accumulation of water.

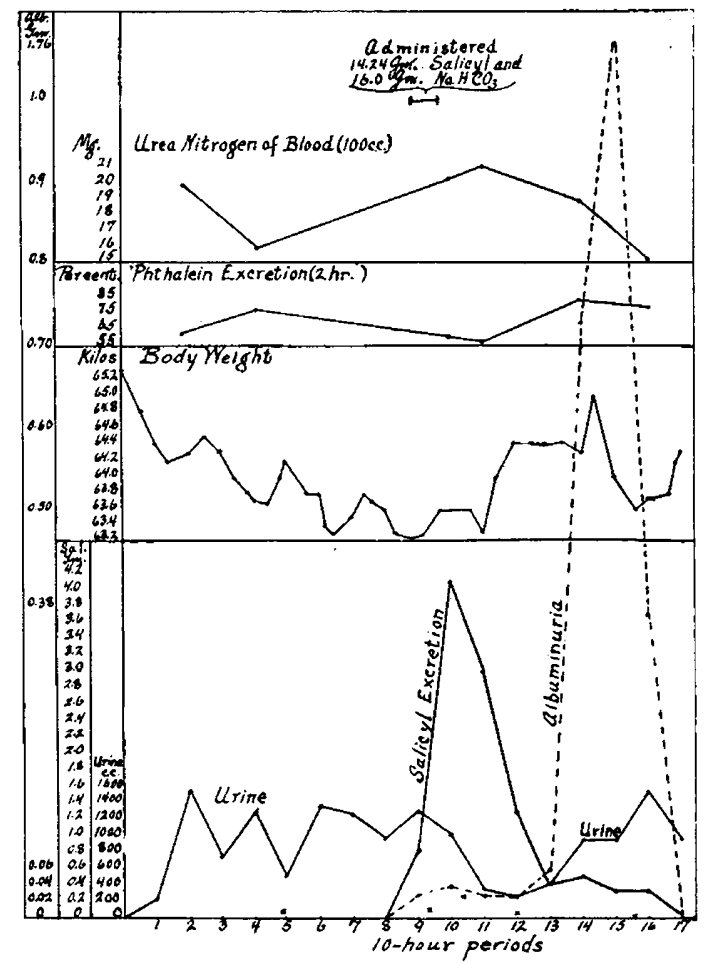

Chart 9.-Patient 30 (H. M.)

However, the following observation made by Sollmann and Pilcher" tends to exclude direct action on the tissues, or at least so far as local edema is concerned, and therefore lends support to the renal factor. In connection with the study of a series of agents which give rise to endermic reactions characterized by hyperemia, swelling (wheal formation), etc., the application of sodium salicylate to the abrazed skin does not give rise to local edema or urticaria such as is unmistakably produced by a large number of other drugs. The concentration used

6. Sollmann and Pilcher: Jour. Pharmacol. and Exper. Therap., 1917, 9, 309. 
by Sollmann and Pilcher is much higher than the concentration of salicyl we have found in the blood even after the largest doses used by $u$ s. There is no reason to believe that salicyl would be more effective in smaller than in larger concentrations.

5. Effect of the Administration of Sodium Bicarbonate. - If the edema produced by the administration of salicylate is of the same nature as the salt edema of Widal, it could conceivably be accentuated by the administration of such a salt as sodium bicarbonate. It has also been observed that the administration of large doses of bicarbonate can cause edema. This also gave us the opportunity to test the claim that is made by some, namely, that edema is due to the production of acid, and the rational therapy indicated consists of the administration of alkali.

Sodium bicarbonate together with salicylate was administered to Patients 27, 28, 29 and 30. The urines remained alkaline throughout the experiment, or at least, during the increase in body weight. The phenomena observed under the bicarbonate-salicylate medication as compared with salicylate alone, remained practically unaltered. Of four patients studied, only one (29, Chart 8 ) failed to show an increase in body weight, owing to marked diaphoresis.

Sodium bicarbonate, therefore, has no demonstrable influence on the edema and diminution in renal functional efficiency, albuminuria, anuria, etc., produced by the administration of full therapeutic doses of salicylate.

\section{CONCLUSIONS}

1. The anuria produced by the administration of full therapeutic doses of salicylate is due to retention of water as indicated by an increase in body weight unless modified by diaphoresis.

2. This retention is demonstrable about twenty hours after the start of administration of the salicylate and persists until the salicyl excretion is completed, that is, about eighty hours (three and onethird days).

3. The retention occurs chiefly in the tissues, for no dilution of the blood is demonstrable by estimations of hemoglobin.

4. The edema is accompanied by a diminution in phenolsulphonephthalein excretion together with an accumulation of urea nitrogen of the blood, and increased excretion of albumin, all of these elements reaching their previous levels with the disappearance of the edema.

5. There is, therefore, a diminution in renal functional efficiency, and since this generally makes its appearance before an actual increase in body weight is demonstrable (edema) and later coincides with it, 
it seems that the renal factor plays an important rôle in the production of the edema.

6. These phenomena are not modified by the administration of sodium bicarbonate together with the salicylate, and in doses sufficient to maintain the urine alkaline.

We wish to express our thanks to Supt. C. H. McFarland and Dr. E. P. Carter, Chief of Medical Service, for extending the use of a special ward without which this and other investigations on salicylate would have been impossible; to Drs. Sheets and Kennedy, interns, and Messrs. T. W. Thoburn, M. E. Fulk and J. A. West of the third year class for various services rendered in connection with the work. 